\title{
Sedimentation of a colloidal monolayer down an inclined plane
}

\author{
Brennan Sprinkle, ${ }^{1}$ Sam Wilken $\odot,{ }^{2,3}$ Shake Karapetyan, ${ }^{4,3}$ Michio Tanaka, ${ }^{5,3}$ Zhe Chen $\odot,{ }^{1}$ \\ Joseph R. Cruise $\odot,{ }^{6,1}$ Blaise Delmotte $\odot,{ }^{7}$ Michelle M. Driscoll, ${ }^{8}$ \\ Paul Chaikin, ${ }^{3}$ and Aleksandar Donev ${ }^{1, *}$ \\ ${ }^{1}$ Courant Institute of Mathematical Sciences, New York University, New York, New York 10012, USA \\ ${ }^{2}$ Materials Department, University of California, Santa Barbara, California 93106, USA \\ ${ }^{3}$ Center for Soft Matter Research, Department of Physics, New York University, New York 10003, USA \\ ${ }^{4}$ Research Center on Stability, Instability, and Turbulence, New York University Abu Dhabi, \\ Abu Dhabi, United Arab Emirates \\ ${ }^{5}$ Department of Physics and Astronomy, University of Pennsylvania, Philadelphia, Pennsylvania 19104, USA \\ ${ }^{6}$ Department of Physics, Washington University, St. Louis, Missouri 63130, USA \\ ${ }^{7}$ LadHyX, CNRS, Ecole Polytechnique, Institut Polytechnique de Paris, 91120 Palaiseau, France \\ ${ }^{8}$ Department of Physics and Astronomy, Northwestern University, Evanston, Illinois 60208, USA
}

(Received 30 November 2020; accepted 16 February 2021; published 11 March 2021)

\begin{abstract}
We study the driven collective dynamics of a colloidal monolayer sedimenting down an inclined plane. The action of the gravity force parallel to the bottom wall creates a flow around each colloid, and the hydrodynamic interactions among the colloids accelerate the sedimentation as the local density increases. This leads to the creation of a universal "triangular" inhomogeneous density profile, with a traveling density shock at the leading front moving in the downhill direction. Unlike density shocks in a colloidal monolayer driven by applied torques rather than forces [Phys. Rev. Fluids 2, 092301(R) (2017)], the density front during sedimentation remains stable over long periods of time even though it develops a roughness on the order of tens of particle diameters. Through experimental measurements and particle-based computer simulations, we find that the Burgers equation can model the density profile along the sedimentation direction as a function of time remarkably well, with a modest improvement if the nonlinear conservation law accounts for the sublinear dependence of the collective sedimentation velocity on density.
\end{abstract}

DOI: 10.1103/PhysRevFluids.6.034202

\section{INTRODUCTION}

The dynamics of active and driven colloidal suspensions is interesting not only because of its inherent out-of-equilibrium nature, but also because it is often dominated by collective effects leading to the formation of large-scale structures and flows. In the Stokes (overdamped) limit relevant to colloids, the hydrodynamic interactions between the particles are long ranged and strongly depend on the presence of nearby boundaries such as confining walls. While self-propelled colloids (microswimmers) are of great interest, externally driven colloids present a simpler system to analyze and study both analytically and via computer simulations. In particular, the only many-body and long-ranged interactions in driven suspensions are hydrodynamic interactions created by the generated solvent flow. While confining walls generally screen hydrodynamic interactions to be less long ranged than in bulk suspensions, the nature of the hydrodynamic flows generated by the activity or external driving mechanism crucially affects the resulting collective behavior.

*donev@courant.nyu.edu 
In prior work [1-5], some of us studied the collective behavior of microrollers: magnetic colloids sedimented above a bottom floor (wall) in an external magnetic field rotating around an axis parallel to the wall. Each spinning particle propels itself parallel to the wall, but the collective motion in a nondilute suspension is much faster than that of an isolated colloid. For microrollers, the driving mechanism is an applied torque, and the flow field created by a single particle corresponds to a rotlet above a no-slip wall [6,7]. This flow advects other particles both in the vertical direction (away or toward the wall), as well as in the transverse directions parallel to the wall. The resulting collective dynamics is surprisingly rich even at moderate densities, for which in the absence of the external drive the colloids would form a single monolayer with in-plane packing fractions $\phi \leqslant 0.5$. Uniform microroller suspensions develop a two-layer structure with a slow bottom layer and a fast top layer [5], and nonuniform suspensions develop traveling-wave density shocks [4] that are unstable to transverse perturbations [3], leading to a fingering instability that can create stable motile clusters of colloids (critters) held together entirely by hydrodynamic interactions [1].

For the case of a nonuniform suspension of microrollers, the formation and dynamics of a density front can be described by a nonlocal conservation law, and the front has a finite width that is proportional to the typical height of the particles above the wall [4]. Here, we study a similar system of colloids sedimented above a bottom wall but now apply a force, rather than a torque, to drive collective dynamics. This can easily be accomplished in the laboratory simply by tilting the bottom wall at an angle $\theta$ and letting the colloids sediment down the inclined plane. The flow field created by a single particle corresponds to a Stokeslet above a no-slip wall [6,7], and advects other particles primarily in the direction of the motion. As we demonstrate, the resulting collective density dynamics can be modeled rather accurately by a local conservation law that can be approximated by a Burgers equation. This leads to sharp density fronts in the form of propagating shock solutions of the inviscid Burgers equation. Similar Burgers-like shocks have been observed for driven and active suspensions confined in a narrow slit channel (top and bottom walls) [8-11], but a crucial difference is that the local flow field around a particle in these cases is quasi-two-dimensional and corresponds to the flow created by a potential dipole. While colloidal diffusion in a flat monolayer [12] and sedimentation in a vertical channel [13] have been studied both using simulations and experiments, to our knowledge the sedimentation of colloids down an inclined plane has yet to be studied. Carpen and Brady studied theoretically the sedimentation of a colloidal suspension in a thicker slit channel and predicted a transverse instability created by unstable stratification due to shear-induced particle migration [14]. Here, we study a monolayer sedimenting above a single wall and find different dynamics that we study by a combination of experiments, computer simulations, and theory.

We begin by discussing the problem theoretically at a mean-field continuum level in Sec. III D 3, and then present results from experiments and particle-based simulations and compare them to the predictions of the theory in Sec. III.

\section{BURGERS MODEL}

We consider a monolayer of spherical colloids of radius $a$ sedimented above a bottom wall tilted at angle $\theta$, as illustrated in Fig. 1. We take the $x$ axis to be in the direction of the gravitational acceleration $g \sin \theta$, and the $z$ axis to be in the direction perpendicular to the wall and pointing away from the gravitational pull $\sim g \cos \theta$. Here, we consider the quasi-one-dimensional problem where initially the colloids are uniformly distributed in the unbounded (in the theory, bounded at millimeter scales in the experiments) or periodic (in simulations) $y$ direction, and in the $x$ direction the colloids are initially contained in the finite interval $\left[x_{b}(t=0), x_{f}(t=0)\right]$. We wish to quantify the collective dynamics as the colloids sediment downhill as time $t \geqslant 0$ elapses. Specifically, we will average along the $z$ direction and consider the coarse-grained number density of colloids $\rho(x, y, t)$ in the $x y$ plane (parallel to the bottom wall). Although estimates of the Péclet number $\mathrm{Pe}=a V_{\text {avg }}\left[k_{B} T /(6 \pi \eta a)\right]^{-1} \sim 10$ (where $V_{\text {avg }} \in[0.1,0.3]$ is estimated based on Fig. 3 ) are not high, we will neglect in-plane diffusion in our continuum models. Also, we will for now assume there is 


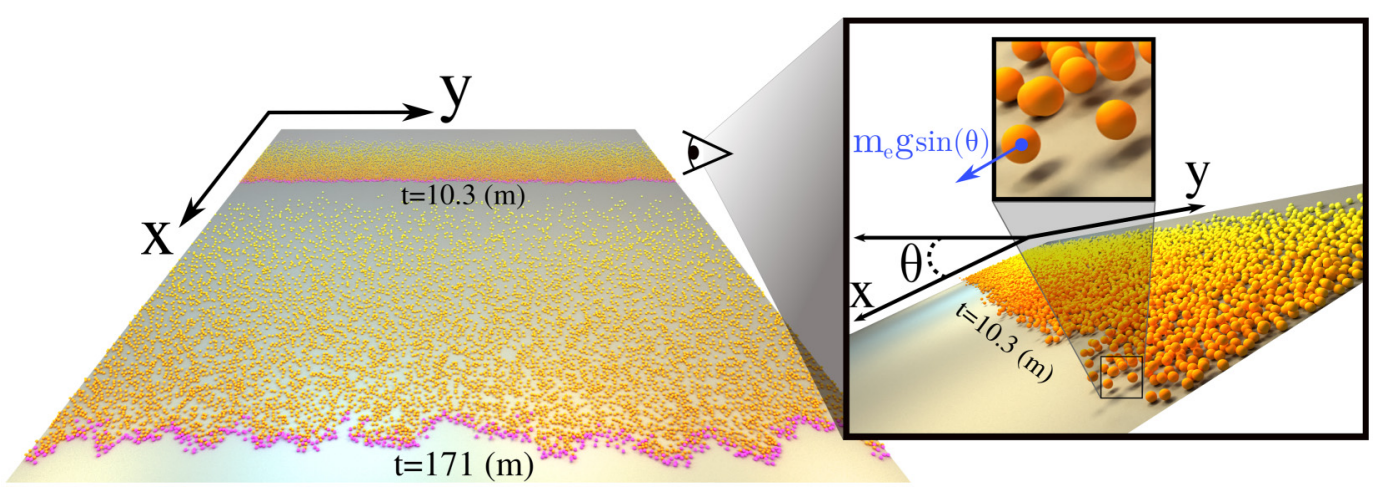

FIG. 1. The left panel shows a frontal view of two snapshots from a typical simulation of a particle suspension sedimenting down an inclined plane. We show the particles at two separate times: the first (top) at a time $t=10.3 \mathrm{~min}$ shortly after the particles have begun to sediment down the plane, and the second at $t=171 \mathrm{~min}$ when the triangular density profile is well formed (see text). The front of the suspension is highlighted by magenta particles, visually emphasizing the coarsening of the density front in time (see Sec. III D 1). The right panel shows a side view of the suspension at $t=10.3 \mathrm{~min}$ along with an inset zoom showing several particles near the front at their typical Brownian height $h_{g}$. An animated version of the left panel is available in the Supplemental Material (SM) [15].

no transverse instability and thus the density remains quasi-one-dimensional, $\rho(x, y, t) \approx \rho(x, t)$, even though the left panel in Fig. 1 shows that this is not exactly true at later times. We discuss the validity of this assumption in more detail in Sec. III D 1, and conclude that the roughness of the front remains small compared to the extent of the density profile in the $x$ direction for all times.

Let us assume that an unbounded uniform suspension with $\rho(x, t) \approx \rho_{0}$ sediments down the plane (in the positive $x$ direction) with collective velocity $v\left(\phi_{0}\right)$, where $\phi=\rho \pi a^{2}$ is the in-plane packing fraction and $a$ is the radius of the colloids. We will use $\phi(x, t)$ instead of $\rho(x, t)$ as a more physically intuitive variable. The collective sedimentation velocity can be estimated as a function of packing fraction experimentally or via computer simulations, as we discuss in Sec. III C. Because particles interact hydrodynamically and advect other particles via the flows they create, the function $v(\phi)$ is quite generally monotonically increasing. For small $\phi \ll 1$ we expect the linear approximation

$$
v(\phi) \approx v_{0}+\frac{S}{2} \phi
$$

to hold, where $v_{0}$ is the sedimentation velocity of an isolated particle, and $S / 2$ is a constant that measures the strength of the hydrodynamic interactions.

It is natural to expect that the density dynamics follows the local conservation law

$$
\frac{\partial \phi}{\partial t}+\frac{\partial}{\partial x}[v(\phi) \phi]=0
$$

which at low densities is expected to be well approximated by the Burgers equation

$$
\frac{\partial \phi}{\partial t}+v_{0} \frac{\partial \phi}{\partial x}+S \phi \frac{\partial \phi}{\partial x}=0
$$

It is important to note that for colloidal microrollers, the local mean-field approximation (2) is not appropriate, and one must take into account the nonlocal nature of hydrodynamic interactions [4]. However, for sedimentation, the hydrodynamic interactions are more local and we expect that the local approximation (2) is suitable; we come back to this question in Sec. IV. 
Adopting the Burgers approximation (2) we can obtain several analytical results. We will set $v_{0}=0$ for convenience since a nonzero self-velocity simply translates the solution to the right by $v_{0} t$. Let us first consider an initial density profile that is a square wave, $\phi(x, t=0)=\phi_{0}$ for $0 \leqslant$ $x \leqslant L$, and zero otherwise. The Burgers equation (3) can be solved analytically, showing that at the front of the square wave there is a propagating shock with position $x_{f}(t)$, with $x_{f}(0)=L$, and at the back of the square wave, which stays fixed at $x_{b}(t)=0$, there is a rarefaction wave. The rarefaction and shock waves meet at time

$$
t_{\text {triang }}=2 \frac{L}{S \phi_{0}}, \quad \text { when } \quad x_{f}\left(t_{\text {triang }}\right)=2 L
$$

After this initial transient, there is a triangular density profile

$$
\phi\left(x, t \geqslant t_{\text {triang }}\right)= \begin{cases}\frac{x}{x_{f}(t)} \sqrt{\frac{2 M}{S t}}=\frac{x}{S t} & \text { if } 0 \leqslant x \leqslant x_{f}(t), \\ 0 & \text { otherwise, }\end{cases}
$$

where the total conserved "mass" is

$$
M=\int \phi(x, t) d x=L \phi_{0},
$$

and the shock wave at the front of the triangle is located at

$$
x_{f}(t)=\sqrt{2 S M t} .
$$

This remarkably simple triangle-wave solution is, in fact, universal and independent of the initial conditions. In particular, it was proven by Lax that any compactly supported initial condition will for long times asymptotically approach the triangle-wave solution (5) [16]. It was further shown by Goodman that shock solutions of the Burgers equation in two dimensions are stable against perturbations in the transverse (i.e., the $y$ ) direction [17]. The reader should note that while the equations of Stokes flow are time reversible, once shocks form the Burgers equation is no longer time reversible ${ }^{1}$ because of the implicit dissipation at the front caused by the neglected diffusion.

\section{RESULTS: SIMULATIONS AND EXPERIMENTS}

We now compare results from experimental measurements and computer simulations to the predictions of the Burgers model. We begin by describing briefly the experimental setup and simulation methodology, and then present results for the collective sedimentation velocity $v(\phi)$ for uniform suspensions, before studying the formation and propagation of Burgers density shocks. For the results presented here we incline the bottom floor at an angle $\theta=45^{\circ}$, though we studied other angles as well and found that the Péclet number controls the layering of the suspension. For larger angles $\left(\theta \gtrsim 60^{\circ}\right)$, at larger packing densities some of the particles get lifted away from the floor and the colloids are not in a monolayer, while for smaller angles the sedimentation velocity is low and diffusion becomes important. For $\theta=45^{\circ}$ and the Péclet numbers considered in this work we find that the suspension remains in a monolayer.

\section{A. Experiments}

We performed sedimentation experiments with colloidal monolayers sedimenting down an inclined plane at angle $\theta=45^{\circ} \pm 3^{\circ}$ placed inside of a custom-built tilting microscope that sets the angle. The colloids are polystyrene spheres (Duke Scientific) of diameter $d=2 a=(4.2 \pm 0.1) \mu \mathrm{m}$ suspended in mixtures of water $\left(\mathrm{H}_{2} \mathrm{O}\right)$ and heavy water $\left(\mathrm{D}_{2} \mathrm{O}\right)$ to modify the gravitational height $h_{g}=k_{B} T /\left(m_{e} g\right)$, where $g$ is the acceleration of gravity, $m_{e}$ is the buoyant mass, and the temperature

\footnotetext{
${ }^{1}$ For example, many initial conditions can reach the same triangle solution (5) after some finite time [16].
} 
$\mathrm{t}=0 \mathrm{~min} \quad \mathrm{t}=9.2 \mathrm{~min}$
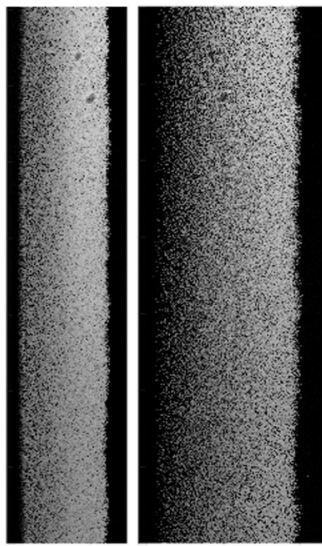

$\mathrm{t}=45.3 \mathrm{~min}$

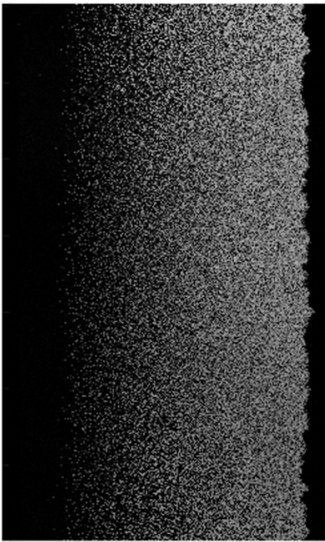

$\mathrm{t}=183.4 \mathrm{~min}$

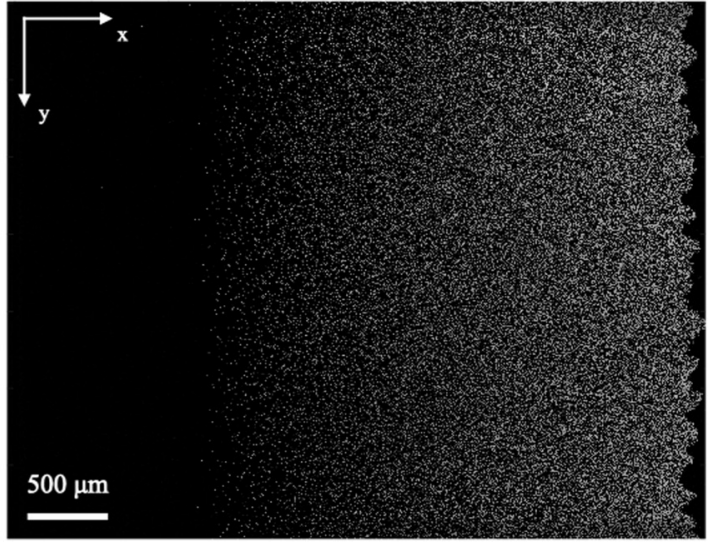

FIG. 2. Microscope images of the density profile for experiment No. 2 at $h_{g}=0.336 \mu \mathrm{m}$ at several times. The scattered light intensity is roughly proportional to the density. As time progresses an inhomogeneous (triangular) density profile develops (higher density on the right, the direction of motion of the front). See Fig. 8 for averages along the $y$ axis, and the middle panel in Fig. 4 for images of the front extracted from this data. An animated version of this figure is available in the SM [15].

$T$ is $(22 \pm 3)^{\circ} \mathrm{C}$. Note that the normal distance between the surface of the particles and the bottom wall is $\sim k_{B} T /\left(m_{e} g \cos \theta\right)$. We use three mixtures with different mass fraction of heavy water: $0 \%$ heavy water $\left(h_{g}=0.250 \mu \mathrm{m}\right), 12.5 \%$ heavy water $\left(h_{g}=0.336 \mu \mathrm{m}\right)$, and $25 \%$ heavy water $\left(h_{g}=0.500 \mu \mathrm{m}\right)$. The solution also contains 50-mM BIS-TRIS (bis (2-hydroxyethyl) amino-tris (hydroxymethyl) methane) to stabilize the particles.

The sample chamber is a rectangular borosilicate capillary tube with dimensions $6 \times 25 \times$ $0.3 \mathrm{~mm}$. The glass surface is coated with polyelectrolyte multilayers, specifically, three layers each of PDADMAC poly(diallyldimethylammonium chloride)(+) and PSS poly(styrene sulfonate)(-) to prevent particle sticking to the glass surface. The colloidal suspensions are loaded into capillaries at a three-dimensional volume fraction $5.0 \times 10^{-4}$ and the sample chamber is centrifuged first along the $z$ axis to rapidly drive particles to the bottom surface, and then centrifuged along the $x$ axis "uphill" to create a densely packed, partially crystallized monolayer with area fraction of $0.7 \pm 0.1$, extending over a distance of approximately 100 particle diameters at the bottom of the capillary. This is the initial condition of all sedimentation instability experiments. Experiments to measure sedimentation velocity as a function of density for uniform suspensions are prepared the same way without the $x$-axis centrifugation.

Sedimentation density profiles are imaged with a custom-built tilting microscope. The camera, objective, sample, and back lighting [530-nm light-emitting diode (LED)] are all mounted together and rotated about a single axis so that prepared monolayers can be imaged while rotated from flat $\theta=0$ (relative to gravity) to an incline at angle $\theta$, while the whole monolayer remains in the focal plane. A photograph of the microscope can be found in the SM [15]. Sample snapshots from the evolution of the monolayer density observed in experiments are shown in Fig. 2. For experiments with a uniform suspension we employ conventional particle tracking techniques [18] to count particles and obtain an accurate estimate of $\phi$.

Large fields of view were needed to accurately measure the density evolution over long periods of time, limiting the accuracy of particle tracking techniques, especially at the highest packing fractions. We therefore use scattered intensity as a proxy for particle density in the $x y$ plane (see Fig. 2). This is not sufficiently accurate to obtain precise measurements of $\phi(x, y, t)$ since the intensity is not strictly linear in the density, and also because of imaging artifacts; this is evidenced in the fact that the total intensity is not conserved and fluctuates by $\sim 10 \%-20 \%$ especially when the 
TABLE I. Parameters (rows) used in the simulations for different gravitational heights (columns) $h_{g}=$ $k_{B} T / m_{e} g$ of the colloidal monolayer.

\begin{tabular}{lccc}
\hline \hline $\mathrm{D}_{2} \mathrm{O}(\%)$ & 0 & 12.5 & 25 \\
\hline$h_{g}=$ & $0.25 \mu \mathrm{m}$ & $0.336 \mu \mathrm{m}$ & $0.5 \mu \mathrm{m}$ \\
$\rho_{f}=$ & $998 \mathrm{~kg} / \mathrm{m}^{3}$ & $1009 \mathrm{~kg} / \mathrm{m}^{3}$ & $1020 \mathrm{~kg} / \mathrm{m}^{3}$ \\
$\eta=$ & $0.95 \mathrm{mPa} \mathrm{s}$ & $0.98 \mathrm{mPa} \mathrm{s}$ & $1.01 \mathrm{mPa} \mathrm{s}$ \\
\hline$L_{y}=$ & $116 \mu \mathrm{m}$ & $106 \mu \mathrm{m}$ & $77 \mu \mathrm{m}$ \\
$N=$ & 2759 & 2592 & 1811 \\
$T_{s}=$ & $5.3 \mathrm{~min}$ & $10.2 \mathrm{~min}$ & $38 \mathrm{~min}$ \\
\hline \hline
\end{tabular}

packing fraction is larger than $\phi \gtrsim 0.4$ (see top of Fig. 8). Near the end of the experiments, when the density is lower, $\phi \lesssim 0.2-0.3$, we are able to locate particles reliably, as confirmed by the fact that the total number of particles is conserved to a few percent. For such low densities we confirm that the scattered light intensity is a good proxy for the packing fraction. The total number of particles is approximately conserved among all Burgers shock experiments since we start with the same amount of stock colloidal solution in each capillary and then add water and $\mathrm{D}_{2} \mathrm{O}$ to change $h_{g}$. The total number of particles $N_{\text {expt }} \approx 78500$ in the viewing area was estimated using particle tracking at later times, with the length of the frame being $L_{y}=3344 \mu \mathrm{m}$, giving the total (conserved) "mass" (6) to be $M_{\text {expt }}=N_{\text {expt }} \pi a^{2} / L_{y} \approx 325 \mu \mathrm{m}$. Knowing the total number of particles (total mass) allows us to rescale the scattered intensity to a packing density, which can then be compared to theoretical and computational predictions.

\section{B. Simulations}

The numerical methods used in our simulations are described in detail in recent work by some of us [5] on the collective dynamics in uniform suspensions of microrollers. Briefly, our lubrication-corrected Brownian dynamics method simulates the translational and rotational dynamics of all particles in the suspension in three dimensions. The method is based on the method of Stokesian dynamics and includes both far-field hydrodynamics (at the Rotne-Prager level [19] without stresslets) as well as semianalytical lubrication corrections due to particles coming close to other particles or the bottom wall. We consistently and efficiently account for Brownian motion, which is essential in the particle simulations in order to set the gravitational height of the particles.

In the simulations we take the radius of the particles $a=2.1 \mu \mathrm{m}$ and the density of the particles is set to $\rho_{p}=1041 \mathrm{~kg} / \mathrm{m}^{3}$, and we fix the temperature at $22^{\circ} \mathrm{C}$. We compute the density of the solvent $\rho_{f}$ from the concentration of $\mathrm{D}_{2} \mathrm{O}$ from standard tables [20]; the values are indicated in Table I. Adding $\mathrm{D}_{2} \mathrm{O}$ to water also changes the viscosity of the solution $\eta$ [21], as indicated in Table I. The steric repulsion potential between the particles and between the particles and the wall is approximated by [5]

$$
\Phi(r)=\Phi_{0} \begin{cases}1+\frac{d-r}{2 a \delta_{\mathrm{cut}} / \ln (10)}, & r<d, \\ \exp \left(\frac{d-r}{2 a \delta_{\mathrm{cut}} / \ln (10)}\right), & r \geqslant d,\end{cases}
$$

where $r$ is the distance from a particle to another particle or to the wall, $d=a\left(1-\delta_{\text {cut }}\right)$ for particle-wall repulsion, and $d=2 a\left(1-\delta_{\text {cut }}\right)$ for particle-particle repulsion. ${ }^{2}$ We set the strength of the repulsion to $\Phi_{0}=4 k_{B} T$ and take $\delta_{\text {cut }}=10^{-2}$ to mimic approximately hard sphere interactions through a "firm potential" (see Sec. 3c of Ref. [5]). To accurately resolve the suspension dynamics

\footnotetext{
${ }^{2}$ Note that while the case $r<d$ should not happen for hard particles, slight overlaps do occur in numerical simulations.
} 
we set the time step size to $\Delta t=4.88 \times 10^{-3}\left(6 \pi \eta a^{3}\right) /\left(k_{B} T\right)=0.2 \mathrm{~s}$. Given this comparatively small $\Delta t$, on the order of hundreds of thousands of time steps are needed to simulate the long timescales observed in our sedimentation experiments, which limits the number of particles we can include in our simulations relative to the experiments.

Our simulations are unbounded in the positive $z$ direction, which is a good approximation to the experiments where $L_{z} / a \approx 150 \gg 1$. For uniform suspensions, we use a domain that is periodic with period $L_{x y}$ in both the $x$ and $y$ directions; we controlled the packing fraction by varying $L_{x y}$ while keeping the number of particles fixed. For Burgers shocks the simulation domain is infinite in the $x$ direction (the direction of motion) and periodic in the $y$ (the spanwise) direction with period $L_{y}$ indicated in Table I; the smallest is $L_{y} / a \sim 37$, which we have confirmed is sufficiently large to make periodic artifacts in the hydrodynamics negligible.

To generate an initial configuration of particles at time $t_{0}$ confined to a region of the $x y$ plane of area $L_{x} \times L_{y}$, we perform Markov chain Monte Carlo equilibrium runs at a constant in-plane packing fraction $\phi_{0}$ with $N_{0}$ particles. For uniform suspensions, $\phi_{0}=\phi$ and we keep all $N=N_{0}=1024$ particles. For Burgers shock simulations, we need to generate an inhomogeneous density profile. Therefore, we first generate a uniform one at the maximum density and then randomly remove particles to reduce the density where needed. Specifically, we take the initial density profile extracted from experiments $\phi_{\text {exp }}\left(x, t_{0}\right), \operatorname{set}^{3} \phi_{0}=1.1 \max \phi_{\text {expt }}\left(x, t_{0}\right)$ and $N_{0}=4000$, and independently and uniformly randomly remove each particle with probability $\left[1-\phi_{\text {expt }}\left(x, t_{0}\right) / \phi_{0}\right]$. The final number of particles $N$ after this rejection step is indicated in Table I.

\section{Uniform suspensions}

Our computational and experimental results for the collective sedimentation velocity $v(\phi)$ at the three different gravitational heights are shown in Fig. 3. The apparent $x$ velocity of the particles is computed over intervals of 1 second (but the results are not sensitive to this choice), using particle tracking in the experiments, and the average velocity is computed for each packing fraction. We see a good agreement between the measured and predicted collective velocities, to within experimental uncertainty. The relationship is roughly linear as in (1). To obtain a more accurate functional form of $v(\phi)$ to use in (2), we fit the simulation data with a rational function (typically with degrees $3 / 2$ but no larger than 5/2, not shown). Since the simulation results for $v(\phi)$ shown in Fig. 3 are sublinear over the range of densities of interest $(\phi<0.6)$, we refer to (2) as the sub-Burgers equation.

\section{Burgers shock waves}

In the remainder of this section we focus our attention to comparing our experimental and simulation results to the prediction of the mean-field sub-Burgers model (2) and its Burgers approximation (3).

\section{Transverse (in)stability}

Before we discuss results for the one-dimensional density profiles $\phi(x, t)$ modeled by Eqs. (2) and (3), we must understand whether the two-dimensional density profiles $\phi(x, y, t)$ are actually effectively one dimensional, that is, whether the density front remains stable to transverse perturbations.

To do this, from the experimental images and binned simulation densities, we extract the position of the density front $x_{f}(y ; t)$ such that the density is essentially zero for $x>x_{f}(y)$; see the left panel of Fig. 1 for an illustration of the particles determined to be at the density front at two different times. The extracted fronts $x_{f}(y ; t)$ are shown in Fig. 4. For a rough comparison, we also perform a simulation at $h_{g}=0.336 \mu \mathrm{m}$ with $N=8000$ particles and a wider domain with periodic

\footnotetext{
${ }^{3}$ The factor of 1.1 gives us a small buffer above the maximum.
} 


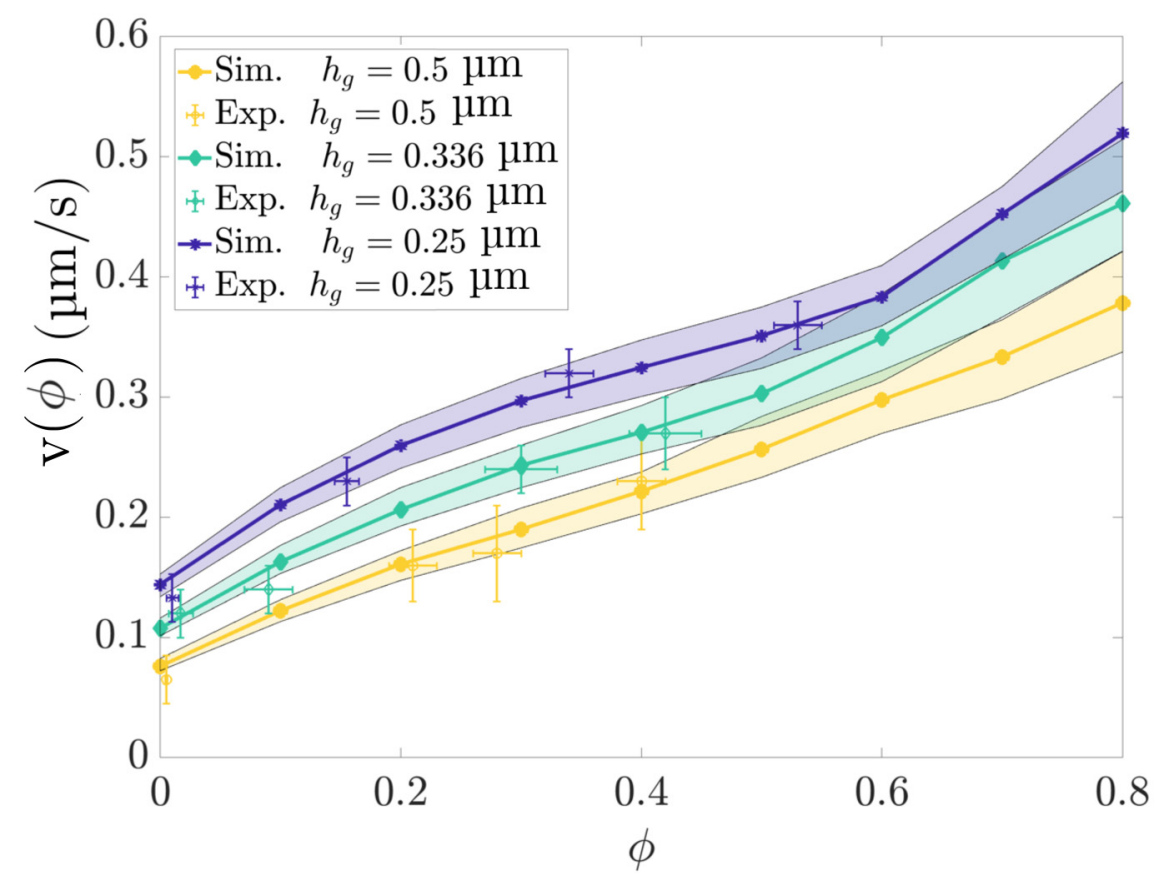

FIG. 3. Collective sedimentation velocity as a function of in-plane packing fraction for each of the three gravitational heights, for both simulations and experiments. Experimental measurements of $v(\phi)$ are shown as markers with error bars of one standard deviation. The solid curve shows data from simulations at $\theta=45^{\circ}$; the boundaries of the shaded region around the simulation data are from simulations with $\theta=42^{\circ}$ (smaller velocity) and $\theta=48^{\circ}$ (larger velocity), in order to account for the experimental uncertainty in the sedimentation angle $\theta=45^{\circ} \pm 3^{\circ}$. In the simulations the colloids remained in a monolayer only for $\phi \lesssim 0.6$.

length $L_{y}=1260 \mu \mathrm{m}$, and a uniform initial packing density of $\phi\left(0 \leqslant x \leqslant L_{x}, y, t\right)=0.6$ where $L_{x}=L_{y} / 8.6$. We show the simulated density front profiles overlayed on top of the experimental ones in the middle panel of Fig. 4, but it should be noted that a direct comparison is not possible because at present we cannot simulate a system of the same dimensions as the experiments due to the very large number of particles in the experiments. Due to the unmatched conditions between the simulation and experiment, the shift in the mean position of the front in the simulations has been scaled by an empirical factor of 2.6 to align the fronts with the experiments for easier visual comparison. This factor is consistent with a rough estimate based on the Burgers timescale (4) assuming the initial condition in experiments is a square wave, $t_{\text {triang }}^{(\text {expt }} / t_{\text {triang }}^{(\text {(sim) }}=\left(L^{(\text {expt })} \phi_{0}^{(\text {(sim) }}\right) /\left(L^{(\text {sim })} \phi_{0}^{(\text {expt })}\right) \approx$ $(500 \mu \mathrm{m} \times 0.6) /(147 \mu \mathrm{m} \times 0.65) \approx 3.1$.

The results in Fig. 4 show that the density front becomes rough over time, however, they do not show the formation of a transverse instability at a precise wavelength, as is the case for microrollers $[2,3]$. Instead, the roughness has a broad range of characteristic length scales [as determined from the correlation function of $\left.x_{f}(y ; t)\right]$ and pinpointing a precise "wavelength" of the roughness is not possible due to the large statistical uncertainty, as discussed in the SM [15].

Importantly, the relative roughness of the density shock appears to reach an approximately constant magnitude, with the front position fluctuating over a range of about $100 \mu \mathrm{m}$ at the final time. To verify this, in Fig. 5 we show the width of the rough profile $\sigma_{f}(t)$, as measured from the standard deviation of $x_{f}(y ; t)$. We normalize the roughness by the length of the density profile $x_{f}(t)-x_{b}(t)$ [predicted to grow as $\sqrt{t}$ by the Burgers model (7)], where henceforth we denote the mean position of the density front with $x_{f}(t)=\left\langle x_{f}(y ; t)\right\rangle$. To estimate the position of the back of 


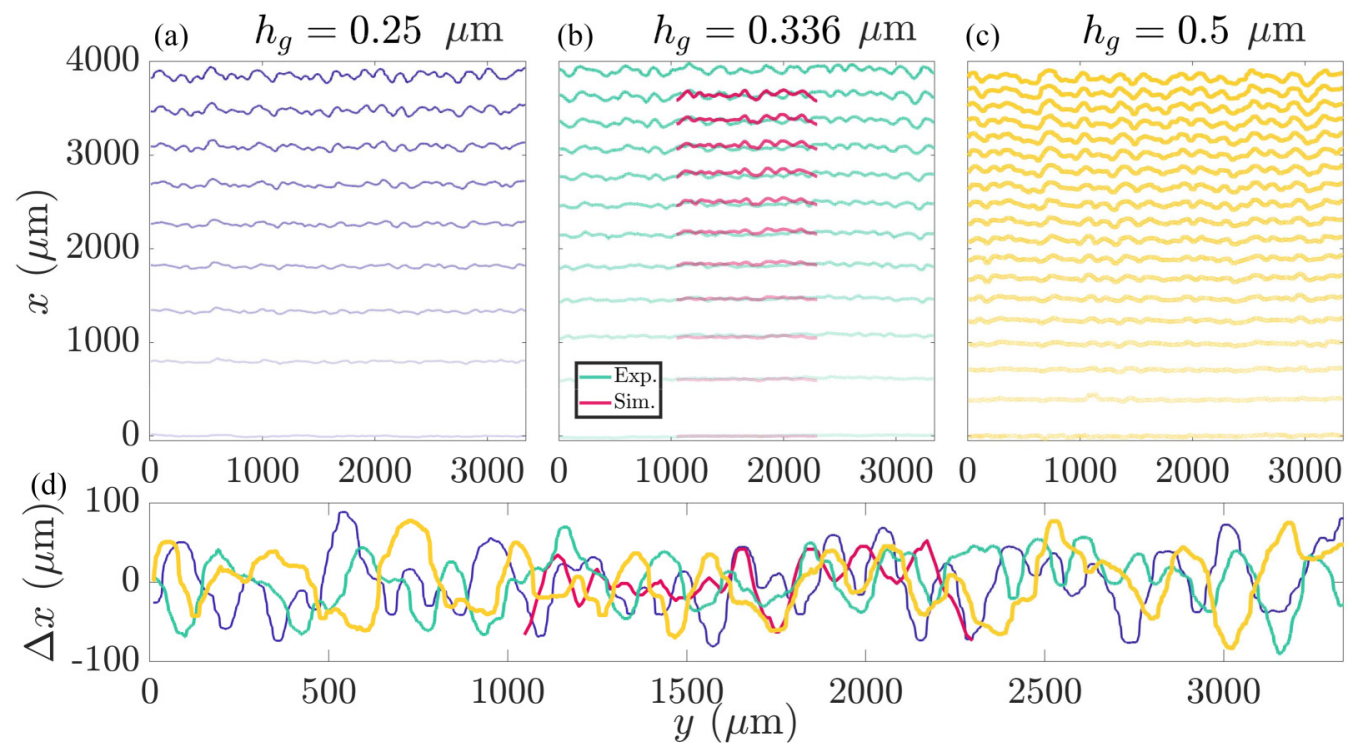

FIG. 4. Position of the density front $x_{f}(y ; t)$ in one of the experiments at each gravitational height. In panels (a), (b), and (c) we overlay snapshots of the experimental density front in time increments of $1000 \mathrm{~s}$, where the opacity of the curves increases with time. (b) also shows the results of a particle simulation (magenta line) we ran for $h_{g}=0.336$ using $N=8000$ particles with initial density $\phi_{0}=0.6$, where the time is scaled by a factor of 2.6 to account for the different total number of particles and domain geometry. (d) overlays the shifted fronts $\Delta x(y ; t)=x_{f}(y ; t)-\left\langle x_{f}(y ; t)\right\rangle$ at the final time for each gravitational height, as well as the simulation results for $h_{g}=0.336$. We see that the scale of the roughness and characteristic "wavelength" of the front are roughly independent of $h_{g}$ and are comparable in experiments and simulations. Further discussion of the characteristic wavelength can be found in the SM [15].

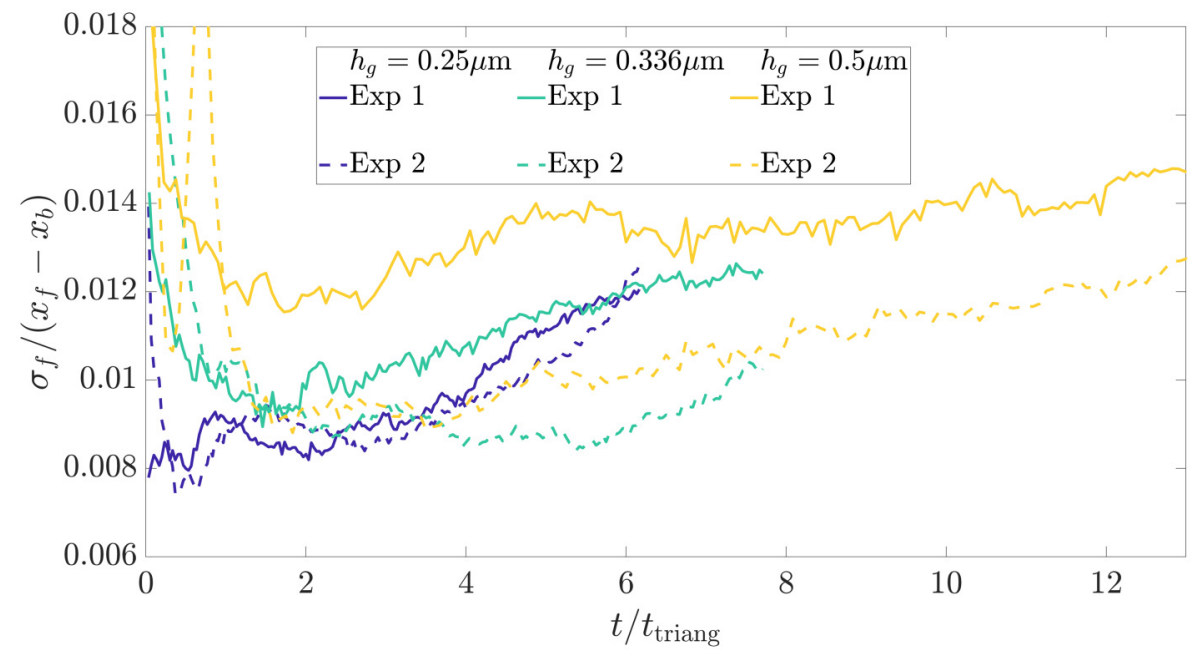

FIG. 5. The scale of the roughness of the density front $\sigma_{f}(t)=\operatorname{std}\left[x_{f}(y ; t)\right]$, normalized by the length of the density profile $x_{f}(t)-x_{b}(t)$, for two experiments at each of the three gravitational heights. Time is scaled by a rough estimate of the time it takes for the density profile to become (approximately) triangular, $t_{\text {triang }}=20 \mathrm{~min}$ [see also (4)]. In the SM [15] we show the temporal evolution of the characteristic wavelength of the fronts. 
the front $x_{b}(t)$, we fit a line to the section of the density profile corresponding to the first fifth of its extent in the $x$ direction, and calculate $x_{b}$ as the intercept of this line. This is reasonably robust except in the initial stages of the experiment when the density profile is far from linear.

We see in Fig. 5 that in all experiments the roughness of the front remains quite small $(\sim 1 \%)$ compared to the length of the density profile, at all times after an initial transient [predicted to be $t_{\text {triang }}$ by the Burgers model, see (4)] during which the triangular density profile predicted by (5) is reached. Therefore, even though the front roughness is large compared to the particle size (as much as 50a), it is still reasonable to approximate the density profiles as effectively one dimensional, $\phi(x, y, t) \approx \phi(x, t)$. Thus, unless otherwise stated, we focus on analyzing the density $\phi(x, t)$ computed by averaging $\phi(x, y, t)$ along the $y$ direction.

\section{Sub-Burgers model}

In this section, we compare the predictions of the sub-Burgers model (2), using the estimated collective sedimentation velocity $v(\phi)$ shown in Fig. 3, to the Burgers approximation (3). Because particle tracking over the length scales and timescales of interest was not possible in the experimental studies of sedimenting Burgers fronts, we cannot do this comparison for the experiments since we cannot accurately measure $\phi(x, t)$ in the experiments over the whole range of time. Instead, we use computer simulations to evaluate the accuracy of the models (2) and (3).

We do our best to make the initial conditions in the simulations mimic the experiments by using the experimental scattered light intensity, scaled using the total number of particles estimated by particle tracking, as a proxy for $\phi(x, t)$. Since this is a particularly bad approximation at the very high packing densities in the initial configuration in the experiments, we skip an amount of time $T_{s}$ in the beginning of each experiment so that the maximum packing density falls to around 0.4 ; the times $T_{s}$ are indicated in Table I. For the particle-based simulations, we generate initial conditions from the experimentally measured $\phi\left(x, T_{s}\right)$. For comparison, we also numerically solve the subBurgers equation (2) using a high-resolution Godunov method [22] starting with $\phi\left(x, T_{s}\right)$ as the initial condition.

The results of our computations are summarized in Fig. 6. We see that the initial condition quickly evolves into an approximately triangular density profile, after which the sub-Burgers model (2) and the Burgers approximation (3) (see Sec. III D 3) both agree quite well with the results from the particle simulations. At early times, when the density profile is not yet triangular, the sub-Burgers equation shows reasonable agreement with the particle simulations, but the Burgers approximation does not, as expected. This is particularly evident in the bottom panel of the figure for $h_{g}=0.5 \mu \mathrm{m}$; similar behavior is seen at earlier times (not shown) for the other values of $h_{g}$

We compare the predictions from our simulations to the experimental measurements in Fig. 7. While we cannot measure the density profile accurately in the first part of the experiments due to the inability to track particles, $x_{f}(t)$ can be determined accurately from the experimental images at all times. In Fig. 7 we compare predictions for the mean front position $x_{f}(t)$ between the experiments, particle simulations, the sub-Burgers model (2), and the Burgers approximation (3). The sub-Burgers model is once again seen to agree with the particle simulations rather well over the whole time interval, for all gravitational heights. At the same time, we observe a moderate $(\sim 10 \%-15 \%)$ difference between the particle simulations and experiments for the smallest gravitational height $h_{g}=0.25 \mu \mathrm{m}$, with the agreement becoming much better for the largest $h_{g}=0.5 \mu \mathrm{m}$. The systematic difference persists even if we start simulations from snapshots of the experiments at later times, when the density is lower and the scattered intensity is a better proxy for the true density. This suggests that there is a (yet unidentified) discrepancy in either the particle interactions (e.g., electrostatics), or the hydrodynamics, between the simulations and experiments. ${ }^{4}$ At the largest $h_{g}$,

\footnotetext{
${ }^{4}$ It should also be noted that a one-to-one comparison is difficult to make because the width $L_{y}$ is much smaller in the simulations to reduce the overall number of particles and thus control the computational effort (e.g., $L_{y} \sim 3.35 \mathrm{~mm}$ is the width of the viewing frame in the experiments, while $L_{y} \sim 100 \mu \mathrm{m}$ in the simulations).
} 

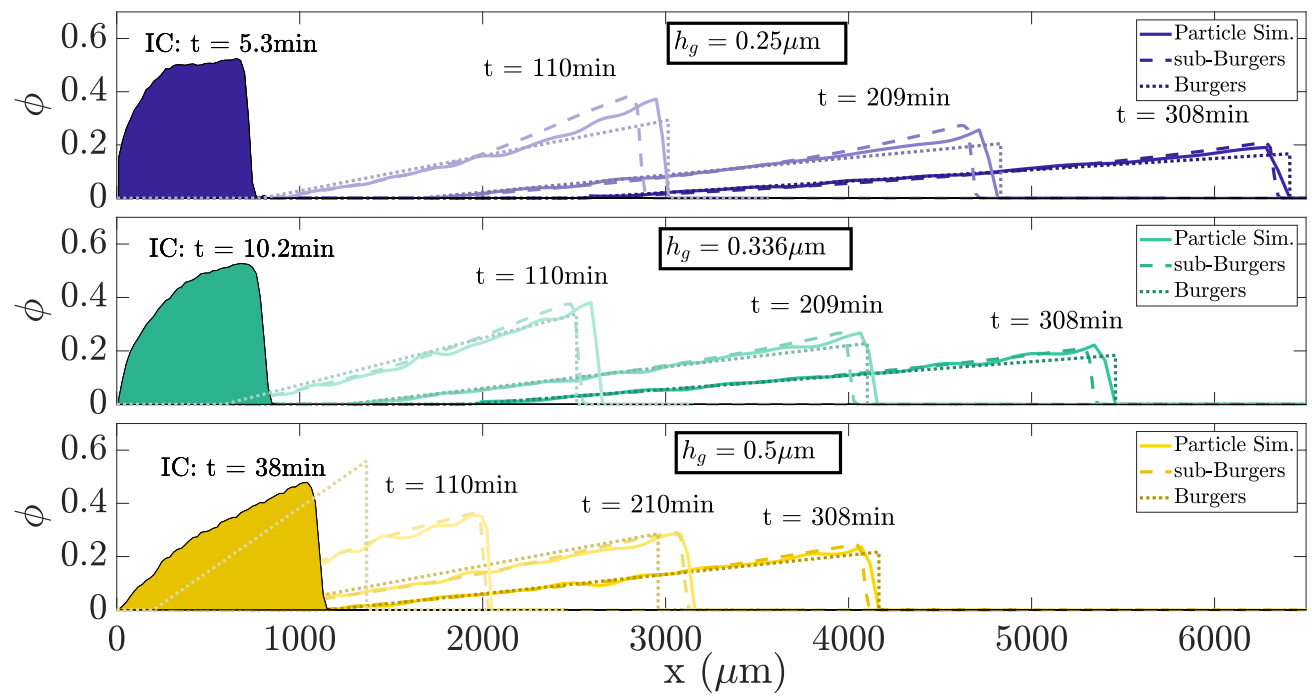

FIG. 6. Evolution of the density profile $\phi(x, t)$ for each gravitational height (increasing from top to bottom). The solid density profile shows the initial condition used in each case, taken from our experimental data after an initial relaxation period (indicated in the figure). Each panel shows the evolution of the density profile in a particle simulation (solid lines, results are reproducible in statistically independent simulations), the subBurgers model (2) (dashed lines), and the Burgers approximation (3) (dotted lines). Evolution of the density profiles in each case is shown using decreasing transparency with increasing time (indicated in the figure). An animated version of this figure is available in the SM [15].

the particles are furthest from each other and the floor, and we expect the direct and hydrodynamic interactions between particles to have less of an impact. We further compare experiments and simulations quantitatively in Sec. III D 3.

\section{Burgers model}

In Sec. III D 2 and, in particular, in Fig. 6, we compared the predictions of the Burgers equation (3) as an approximation to the sub-Burgers equation (2) at late times (when density is low). The sub-Burgers model requires estimating/approximating the collective velocity function $v(\phi)$, and the Burgers approximation further requires estimating the self-velocity $v_{0}=v(\phi=0)$ and the slope $S=2 d v(\phi=0) / d \phi$, which can be difficult to do accurately especially with experimental data. Therefore, in this section we see how well the Burgers equation (3) serves as a model for the simulation and experimental results, without going through $v(\phi)$.

In order to compare our simulation results to the predictions of the Burgers model (3), we fit the mean position of the front $x_{f}(t)$ and the back of the front $x_{b}(t)$ to the theoretical prediction (7), accounting also for the fact that $v(\phi=0)$ is nonzero,

$$
\begin{gathered}
x_{b}(t)=v_{0} t+x_{0}, \\
x_{f}(t)-x_{b}(t)=\sqrt{2 S M\left(t-t_{0}\right)},
\end{gathered}
$$

where $v_{0}=v(\phi=0)$ is the numerically estimated self-velocity of an isolated particle (see Table II), $S=2 d v(\phi=0) / d \phi$ is estimated from the slope of the rational fit to the simulation data shown in Fig. 3 (see Table II), and the total mass $M=N \pi a^{2} / L_{y}$. To account for the fact that the initial condition is not a square wave, we obtain the time shift $t_{0}$ and the position shift $x_{0}$ from $x_{f}\left(t_{f}\right)$ and $x_{b}\left(t_{f}\right)$ at the final time $t_{f}$. We remind the reader that the Burgers equation predicts that any compactly 

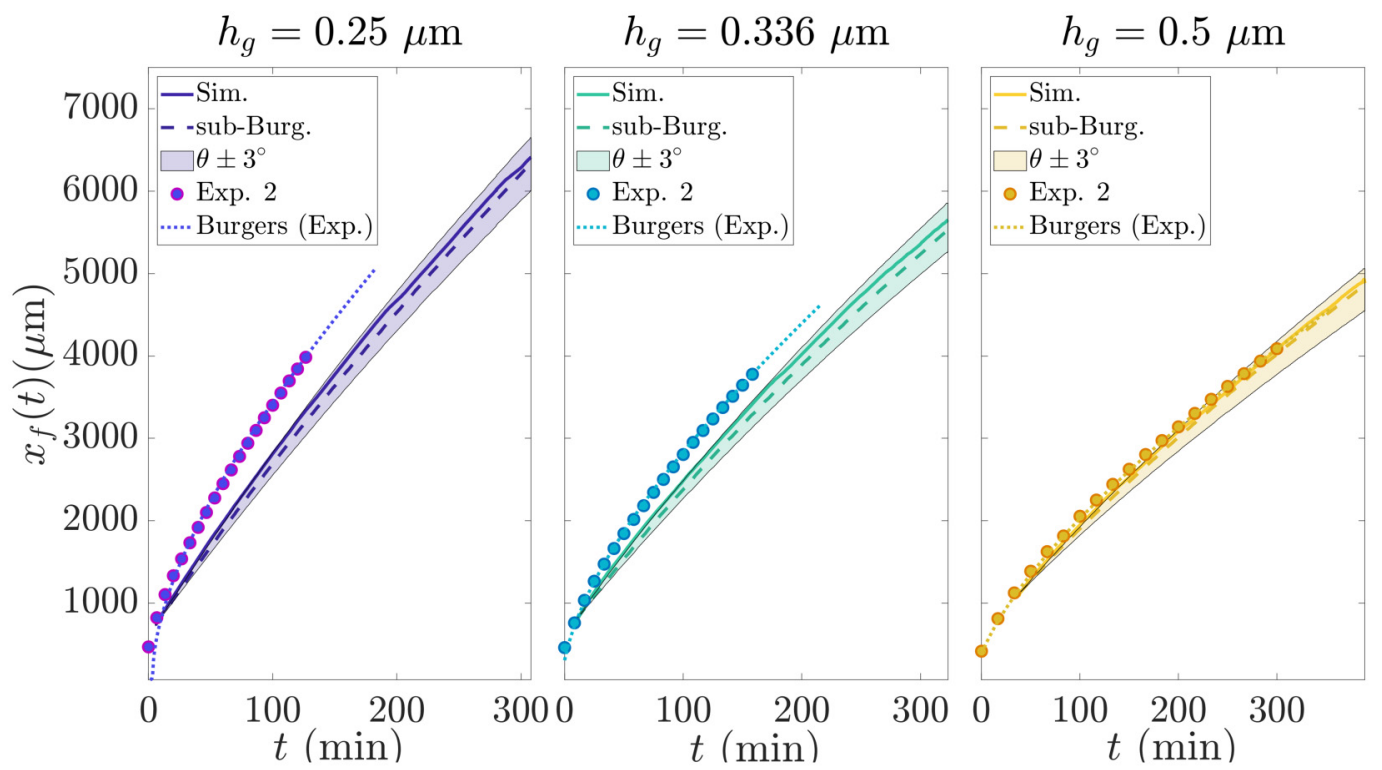

FIG. 7. Mean position of the density front $x_{f}(t)$ for each of the three gravitational heights (increasing from left to right). The experimental results (experiment No. 2) are shown with symbols (circles), and the Burgers fit to the experimental data (see Sec. III D 3 for details) is shown with a dotted line. We only show one of the experiments but the difference between experiments is smaller than the symbol size. The results of particle simulations are shown with a solid line along with corresponding shaded regions bounded by the results obtained when the angle $\theta$ was changed by $\pm 3^{\circ}$, which is roughly the experimental uncertainty in setting the microscope orientation. The predictions of the sub-Burgers model (2) are shown with a thick dashed line of the same color as the particle results.

supported initial condition will asymptotically approach a triangle wave (5) with a time and space shift that depends on the initial conditions.

In Table II we compare the parameter $S$ and the self-velocity $v_{0}$ in the Burger's model (3) between experiments and simulations. In the experiments, we can unambiguously and accurately track the mean front position $x_{f}(t)$ over all times, while the estimates for the end of the profile $x_{b}(t)$ are less reliable and more noisy, especially at early times. Nevertheless, we estimate $v_{0}$ by fitting the experimental $x_{b}(t)$ to (9). The results, shown in Table II, demonstrate that $v_{0}$ is quite consistent between experiments and simulations. To obtain $S, t_{0}$, and $x_{0}$, we match the experimental data to Eqs. (9) and (10) at later times, when the triangular density profile is well formed, by restricting the analysis to the time interval $t_{i}<t<t_{f}$ after the maximum of the estimated density is sufficiently

TABLE II. Fitted or computed values of the parameters $S$ and $v_{0}$ in units of $\mu \mathrm{m} / s$ in the Burgers model (3). For the particle simulations, the values are computed from the simulation data shown in Fig. 3 (see text). For two independent experiments, the value of $S$ is obtained from the experimental data shown in Fig. 7 over the time period where $\phi(x, t)<0.4$ (see text). We also show estimates of $v_{0}$ obtained by fitting the experimental $x_{b}(t)$ to $(9)$.

\begin{tabular}{lccc}
\hline \hline$h_{g}(\mu \mathrm{m})$ & 0.25 & 0.336 & 0.5 \\
\hline Particle sim. & $S=1.33, v_{0}=0.144$ & $S=1.11, v_{0}=0.108$ & $S=0.85, v_{0}=0.07$ \\
Expt. No. 1 & $S=1.61, v_{0}=0.154$ & $S=1.24, v_{0}=0.12$ & $S=0.732, v_{0}=0.0723$ \\
Expt. No. 2 & $S=1.67, v_{0}=0.167$ & $S=1.29, v_{0}=0.112$ & $S=0.766, v_{0}=0.0653$ \\
\hline \hline
\end{tabular}



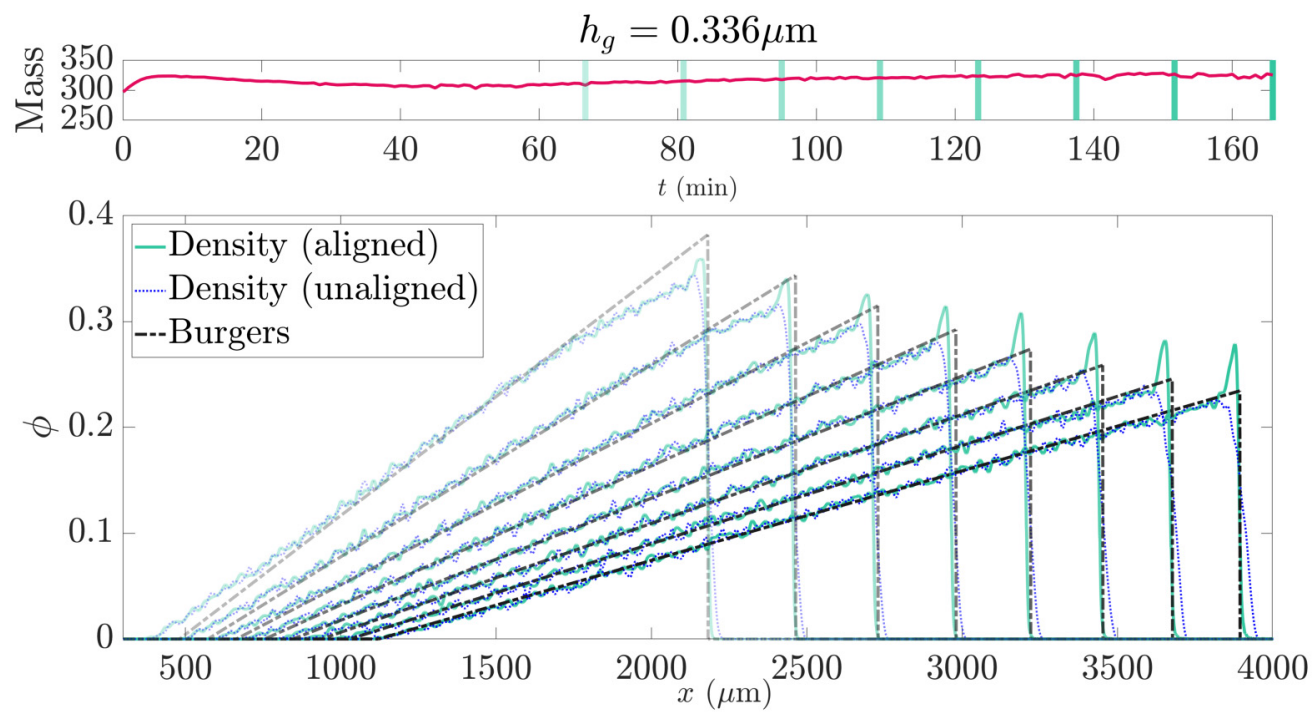

FIG. 8. The top panel shows the total mass of the density profile $M=\int \phi(x, t) d x$ for experiment No. 2 at $h_{g}=0.336$, as measured by scattered intensity rescaled to be in units of particle density based on the estimated total number of particles $N_{\text {expt }}$. The mass remains constant to within $5 \%$ after about $t_{i}=70.8 \mathrm{~min}$, so we compare the experiment data to the Burgers model for $t>t_{i}$. The bottom panel compares snapshots in time of the Burgers model (5) along with the experimental measurements of the density profile. The curves have been colored to increase in opacity as time increases, and the times of each snapshot are indicated by vertical bars in the top panel using a corresponding transparency. The experimental measurements of the density profile have been averaged along the $y$ direction either without (labeled "unaligned") or with alignment (labeled "aligned") of the front $x_{f}(y ; t)$ (see text). An animated version of this figure is available in the SM [15].

low for the Burgers approximation to be apply, $\max \phi\left(x, t_{i}\right)<0.4$. We first determine $x_{0}$ from $x_{b}\left(t_{f}\right)$, since the scattered intensity is the best proxy for the density at the final time, using the self-velocity $v_{0}$ estimated from simulations (see Table II) in order to avoid using the less reliable $x_{b}\left(t_{i}\right)$. Then, we compute $S$ and $t_{0}$ from $x_{f}\left(t_{i}\right)$ and $x_{f}\left(t_{f}\right)$. The resulting Burgers prediction (10) with the estimated parameters is shown in Fig. 7, and is in excellent agreement with the experimental data over nearly the whole time interval. The experimental estimates of the parameter $S$ in Table II show the same qualitative trends with gravitational height, but an imperfect quantitative agreement, between simulations and experiments, as already seen in Fig. 7.

Finally, in Fig. 8 we compare the Burgers prediction (5) and (7) for the density profile to experimental data. For this comparison, we focus on experiment No. 2 at $h_{g}=0.336$, for which the total scattered intensity (proportional to the total mass $M$ ) was constant for $t_{f} \geqslant t>t_{i}=70.8$ min to within 5\%, suggesting that the scattered intensity was a good proxy for $\phi\left(x, t>t_{i}\right)$. The scattered intensity has a small (at most couple of percent of max intensity) constant background intensity (varying slowly over time) both in front of and behind the support of $\phi$. To compute the mass of the density profiles we offset $\phi(x, t)$ to remove the estimated background intensity, and match $M=M_{\text {expt }}$ at the final time $t_{f}$. We estimate the position of the back $x_{b}\left(t_{f}\right)$ by first estimating the position of the front $x_{f}\left(t_{f}\right)$ and then finding the value of $x_{b}\left(t_{f}\right)$ that minimizes the $L_{2}$ error between $\phi\left(x, t_{f}\right)$ and a triangle of area $M$ extending from $x_{f}\left(t_{f}\right)$ to $x_{b}\left(t_{f}\right)$. Using the experimental data for ${ }^{5}$

\footnotetext{
${ }^{5}$ We note that the experimental $x_{b}\left(t>t_{i}\right)$ was in excellent agreement with the theoretical prediction (9) and the value of $v_{0}$ extracted from the simulations (see Table II).
} 
$x_{b}\left(t_{f}\right), x_{f}\left(t_{f}\right)$, and $x_{f}\left(t_{i}\right)$ and Eqs. (9) and (10), we estimate $t_{0}=6.8 \mathrm{~min}$ and $S=1.24$ (compare to data in Table II). In Fig. 8 we compare the theoretical prediction for the triangular density profile (5) with the extracted parameters to the experimental density profile $\phi(x, t)$ obtained by averaging the experimentally measured $\phi(x, y, t)$ along $y$, and subtracting the offset due to the nonzero background intensity. We also compute the average density along $y$ by first shifting the profile $\phi(x, y, t)$ for each $y$ in the $x$ direction by $x_{f}(y ; t)-\left\langle x_{f}(y ; t)\right\rangle$. By construction, this shifting preserves the mean and therefore the aligned density profile has the same mean front position $x_{f}(t)$. The alignment does however allow us to examine the variation in density along the $x$ direction at scales comparable to the particle size, which is not possible with the unaligned profiles because of the smearing caused by the roughness of the front.

We observe in Fig. 8 that both the aligned and unaligned density profiles exhibit the expected triangular shape, and are in reasonable agreement with the Burgers prediction. The aligned data show a sharp density front, but also exhibit a small peak at the front. Numerical simulations suggest that right at the front, the particles get lifted above the floor to heights larger than $h_{g}$, and can therefore reach higher in-plane packing densities. The height of the particles at the front depends on the balance between the self-induced motion due to gravity (pulling them toward the wall) and the upward flow away from the wall, induced by the force acting on the particles behind the front. This effect is more pronounced for larger incline angles $\theta$, and for sufficiently large $\theta$ we find that the particles do not remain in a monolayer, especially near the density maximum at the front.

\section{CONCLUSIONS}

We examined the driven evolution of the planar density profile of a colloidal monolayer sedimenting down an inclined plane, using experiments and simulations. We found that, starting from an approximately constant density in a stripe of finite width, the monolayer develops an inhomogeneous triangular density profile with a shock front at the front edge. At the same time, the front becomes rough, but the relative roughness saturates to an approximately constant amplitude and remains stable. We found that a simple one-dimensional sub-Burgers equation predicts the shape of the density profile well over all times, and a Burgers approximation is accurate at later times when the density is sufficiently low. These simple models only require as input the collective sedimentation velocity of a uniform suspension at a given density, which can be obtained easily in either experiments or simulations. The Burgers equation can be solved analytically and only requires a single input, the slope of the collective velocity as a function of density. We found a modest but systematic difference in the Burgers parameters estimated from fitting experimental data and from simulations, perhaps attributable to unaccounted interactions between the particles and the particles and the floor.

There are many avenues for improving the simple Burgers model we focused on in this work, to try to improve the match between theory, simulations, and experiments. For example, since the roughness of the density front is large compared to the particle size, a natural step would be to consider a two-dimensional conservation law instead of a one-dimensional one. However, the twodimensional Burgers equation is stable to transverse perturbations [17] and would not develop the roughness we observe.

For colloidal microrollers, the local mean-field approximation (2) is not appropriate. Assuming the colloids lie approximately in a plane at height $h$ above the bottom floor, it was shown in Ref. [4] that one should instead use a nonlocal conservation equation

$$
\frac{\partial \rho}{\partial t} \approx-\frac{\partial\left[\rho\left(K_{\text {roll }}^{(h)} * \rho\right)\right]}{\partial x},
$$

where the star denotes convolution with a translationally invariant kernel,

$$
K_{\mathrm{roll}}^{(h)}(x) \sim h x^{2} /\left(x^{2}+4 h^{2}\right)^{2}
$$


that describes the flow field created by a unit rotlet above a no-slip plane [6,7]. The kernel $K_{\text {roll }}^{(h)}(x)$ is zero at the origin, which shows that the collective motion arises due to nonlocal hydrodynamic interactions of particles at distances $O(h)$ rather than neighboring particles. However, the Blake solution for a Stokeslet above a no-slip plane [6,7] can be used to show that the corresponding kernel for sedimentation,

$$
K_{\mathrm{sed}}^{(h)}(x) \sim 4 h^{2} \frac{3 x^{2}+4 h^{2}}{\left(x^{2}+4 h^{2}\right)^{2}}+\ln \left(1+\frac{4 h^{2}}{x^{2}}\right),
$$

is singular at and peaked around the origin, which suggests that collective motion arises primarily due to local hydrodynamic interactions among neighboring particles. It is therefore reasonable to expect that the local approximation (2) is reasonable for sedimentation. ${ }^{6}$

Since we know that the hydrodynamic interactions driving the collective dynamics are nonlocal and the front is rough, it is natural to consider (two-dimensional) nonlocal conservation laws. However, we find that steric repulsion has to be accounted for in such models because otherwise the density develops unphysically large peaks near the front. This is not straightforward to do and would lead to nonlinear nonlocal conservation laws which could not be solved numerically using standard methods (in fact, performing the particle simulations is likely just as fast and simpler), nor would they lead to the physical insight that the simple Burgers model does. In the end, the success of the Burgers equation in describing the collective density dynamics is unexpected but in large part owed to its simplicity and to the universal nature of Burgers shocks at long times [16].

Our work naturally leads to a number of directions for future explorations. Here, we considered the sedimentation of a monodisperse colloidal monolayer. Introducing polydispersity (either continuous or a binary mixture) may lead to a number of phenomena not captured by the simple Burgers equation. For example, it is an open question whether there will be some form of segregation of the particles by size along the density profile, or phase separation or clumping (as predicted by certain lattice models of sedimenting crystals [23]). Nonspherical colloids such as rods would introduce a novel dimension to the problem coupling orientation and density fields, and may also lead to different dynamics than the one studied in this work.

\section{ACKNOWLEDGMENTS}

We thank the anonymous referees for some of the suggestions regarding directions for future explorations. We thank Wenjun Zhao for sharing with us her code for a high-resolution advection solver in one dimension [22], and for improving the handling of limiting. We also thank Jonathan Goodman for helpful discussions regarding the Burgers equation. This work was supported primarily by the MRSEC Program under Award No. DMR-1420073. Additional funding was provided by the National Science Foundation under Award No. CBET-1706562. B.S. and A.D. were supported by the National Science Foundation via the Research Training Group in Modeling and Simulation under Award No. RTG/DMS-1646339. B.S. and A.D. also thank the NVIDIA Academic Partnership program for providing GPU hardware for performing the simulations reported here. P.C. was partially supported by NASA under Grant No. NNX13AR67G S017.

\footnotetext{
${ }^{6}$ In fact, our attempts to solve the nonlocal equation (11) with the kernel $K_{\text {sed }}^{(h)}(x)$ (or the corresponding twodimensional generalization) numerically indicate that the nonlocal equation develops singular solutions where all the particles clump together at the shock front reaching unphysically high densities in finite time. This unphysical behavior arises because steric repulsion is not accounted for in the simple model (11); it is not straightforward to add particle repulsion in continuum models.
} 
[1] M. Driscoll, B. Delmotte, M. Youssef, S. Sacanna, A. Donev, and P. Chaikin, Unstable fronts and motile structures formed by microrollers, Nat. Phys. 13, 375 (2017).

[2] F. B. Usabiaga, B. Delmotte, and A. Donev, Brownian dynamics of confined suspensions of active microrollers, J. Chem. Phys. 146, 134104 (2017); software available at https://github.com/ stochasticHydroTools/RigidMultiblobsWall.

[3] B. Delmotte, A. Donev, M. Driscoll, and P. Chaikin, Minimal model for a hydrodynamic fingering instability in microroller suspensions, Phys. Rev. Fluids 2, 114301 (2017).

[4] B. Delmotte, M. Driscoll, P. Chaikin, and A. Donev, Hydrodynamic shocks in microroller suspensions, Phys. Rev. Fluids 2, 092301(R) (2017).

[5] B. Sprinkle, E. B. van der Wee, Y. Luo, M. Driscoll, and A. Donev, Driven dynamics in dense suspensions of microrollers, Soft Matter 16, 7982 (2020).

[6] J. R. Blake, A note on the image system for a Stokeslet in a no-slip boundary, Proc. Cambridge Philos. Soc. 70, 303 (1971).

[7] Z. Gimbutas, L. Greengard, and S. Veerapaneni, Simple and efficient representations for the fundamental solutions of Stokes flow in a half-space, J. Fluid Mech. 776, R1 (2015).

[8] N. Champagne, E. Lauga, and D. Bartolo, Stability and non-linear response of 1D microfluidic-particle streams, Soft Matter 7, 11082 (2011).

[9] T. Beatus, T. Tlusty, and R. Bar-Ziv, Burgers Shock Waves and Sound in a 2D Microfluidic Droplets Ensemble, Phys. Rev. Lett. 103, 114502 (2009).

[10] A. C. H. Tsang and E. Kanso, Density Shock Waves in Confined Microswimmers, Phys. Rev. Lett. 116, 048101 (2016).

[11] A. Lefauve and D. Saintillan, Globally aligned states and hydrodynamic traffic jams in confined suspensions of active asymmetric particles, Phys. Rev. E 89, 021002(R) (2014).

[12] S. G. Anekal and M. A. Bevan, Self-diffusion in submonolayer colloidal fluids near a wall, J. Chem. Phys. 125, 034906 (2006).

[13] A. Wysocki, C. P. Royall, R. G. Winkler, G. Gompper, H. Tanaka, A. van Blaaderen, and H. Löwen, Direct observation of hydrodynamic instabilities in a driven non-uniform colloidal dispersion, Soft Matter 5, 1340 (2009).

[14] I. C. Carpen and J. F. Brady, Gravitational instability in suspension flow, J. Fluid Mech. 472, 201 (2002).

[15] See Supplemental Material at http://link.aps.org/supplemental/10.1103/PhysRevFluids.6.034202 for animated versions of many of the figures from the body of this work, as well as a photograph of the experimental setup, and a brief discussion of the characteristic wavelength in the sedimentation front.

[16] P. D. Lax, Hyperbolic Systems of Conservation Laws and the Mathematical Theory of Shock Waves (SIAM, Philadelphia, 1973).

[17] J. Goodman, Stability of viscous scalar shock fronts in several dimensions, Trans. Am. Math. Soc. 311, 683 (1989).

[18] J. C. Crocker and D. G. Grier, Methods of digital video microscopy for colloidal studies, J. Colloid Interface Sci. 179, 298 (1996).

[19] J. W. Swan and J. F. Brady, Simulation of hydrodynamically interacting particles near a no-slip boundary, Phys. Fluids 19, 113306 (2007).

[20] E. Swift, The densities of $\mathrm{D}_{2} \mathrm{O}-\mathrm{H}_{2} \mathrm{O}$ mixtures at 5 and $25^{\circ}$, J. Am. Chem. Soc. 61, 198 (1939).

[21] G. Jones and H. J. Fornwalt, The viscosity of deuterium oxide and its mixtures with water at $25^{\circ} \mathrm{C}$, J. Chem. Phys. 4, 30 (1936).

[22] S. May, A. Nonaka, A. Almgren, and J. Bell, An unsplit, higher-order Godunov method using quadratic reconstruction for advection in two dimensions, Commun. Appl. Math. Comput. Sci. 6, 27 (2011).

[23] R. Lahiri, M. Barma, and S. Ramaswamy, Strong phase separation in a model of sedimenting lattices, Phys. Rev. E 61, 1648 (2000). 